\title{
381.
}

\section{NOTE ON BEZOUT'S METHOD OF ELIMINATION.}

[From the Oxford, Cambridge and Dublin Messenger of Mathematics, vol. II. (1864), pp. 88, 89.]

LET $U, U^{\prime}$ be any two rational and integral functions of $x$ of the same order; to fix the ideas let them be the cubic functions

$$
\begin{aligned}
& U=a x^{3}+b x^{2}+c x+d, \\
& U^{\prime}=a^{\prime} x^{3}+b^{\prime} x^{2}+c^{\prime} x+d^{\prime} .
\end{aligned}
$$

Write

$$
\begin{aligned}
& A=\left|\begin{array}{cc}
U, & U^{\prime} \\
a, & a^{\prime}
\end{array}\right|, \quad P=\left|\begin{array}{cc}
U, & U^{\prime} \\
a, & a^{\prime}
\end{array}\right|, \\
& B=\left|\begin{array}{cc}
U, & U^{\prime} \\
b, & b^{\prime}
\end{array}\right|, \quad Q=\left|\begin{array}{cc}
U & , \\
a x+b, & a^{\prime} x+b^{\prime}
\end{array}\right|, \\
& C=\left|\begin{array}{ll}
U, & U^{\prime} \\
c, & c^{\prime}
\end{array}\right|, \quad R=\left|\begin{array}{ll}
U \quad, & U^{\prime} \\
a x^{2}+b x+c, & a^{\prime} x^{2}+b^{\prime} x+c^{\prime}
\end{array}\right|, \\
& D=\left|\begin{array}{ll}
U, & U^{\prime} \\
d, & d^{\prime}
\end{array}\right|, \quad S=\left|\begin{array}{ll}
U & , U^{\prime} \\
a x^{3}+b x^{2}+c x+d, & a^{\prime} x^{3}+b^{\prime} x^{2}+c^{\prime} x+d^{\prime}
\end{array}\right|,=\left|\begin{array}{ll}
U, & U^{\prime} \\
U, & U^{\prime}
\end{array}\right|,=0,
\end{aligned}
$$

then we have

$$
\begin{aligned}
& P=A, \\
& Q=A x+B, \\
& R=A x^{2}+B x+C, \\
& S=A x^{3}+B x^{2}+C x+D,=0,
\end{aligned}
$$


and thence

$$
\begin{aligned}
& A=P, \\
& B=Q-P x, \\
& C=R-Q x, \\
& D=S-R x,=-R x .
\end{aligned}
$$

Let $\alpha$ be an arbitrary quantity and write

we have it is clear

$$
\square z=\left|\begin{array}{ll}
U \quad & U^{\prime} \\
a \alpha^{3}+b \alpha^{2}+c \alpha+d^{\prime}, & a^{\prime} \alpha^{3}+b^{\prime} \alpha^{2}+c^{\prime} \alpha+d^{\prime}
\end{array}\right| ;
$$

$$
\begin{aligned}
\square & =A \alpha^{3}+B \alpha^{2}+C \alpha+D, \\
& =\alpha^{3} P+\alpha^{2}(Q-P x)+\alpha(R-Q x),=R x, \\
& =\left(\alpha^{3}-\alpha^{2} x\right) P+\left(\alpha^{2}-\alpha x\right) Q+(\alpha-x) R,
\end{aligned}
$$

and thence

$$
\frac{\square}{\alpha-x}=\alpha^{2} P+\alpha Q+R \text {. }
$$

The equations $P=0, Q=0, R=0$ are respectively quadratic equations in $x$, the equations which are used in Bezout's method of elimination; and representing them by

we have

$$
\begin{aligned}
& P=L x^{2}+M x+N,=0, \\
& Q=L^{\prime} x^{2}+M^{\prime} x+N^{\prime},=0, \\
& R=L^{\prime \prime} x^{2}+M^{\prime \prime} x+N^{\prime \prime},=0
\end{aligned}
$$

$$
\left|\begin{array}{lll}
L, & M, & N \\
L^{\prime}, & M^{\prime}, & N^{\prime} \\
L^{\prime \prime}, & M^{\prime \prime}, & N^{\prime \prime}
\end{array}\right|=0
$$

as the equation resulting from the elimination of $x$ from the equations $U=0, U^{\prime}=0$. The foregoing investigation shows that the functions $P, Q, R$ are obtained as the coefficients of $\alpha^{2}, \alpha, 1$ in the development of

$$
\frac{1}{a-x}\left|\begin{array}{ll}
U & U^{\prime} \\
a \alpha^{3}+b \alpha^{2}+c \alpha+d, & a^{\prime} \alpha^{3}+b^{\prime} \alpha^{2}+c^{\prime} \alpha+d^{\prime}
\end{array}\right|
$$

or more generally, taking $U, U^{\prime}$ to be any two functions of the order $n$, that the $n$ functions $P, Q, R, \&$ c. each of the order $n-1$ are obtained as the coefficients of $\alpha^{n-1}, \alpha^{n-2}, \ldots \alpha, 1$ in the development of

$$
\frac{1}{a-x}\left|\begin{array}{cc}
U, & U^{\prime} \\
U_{a}, & U_{a}^{\prime}
\end{array}\right|,
$$

where $U_{a}, U_{a}^{\prime}$ are what $U, U^{\prime}$ become when $x$ is replaced therein by $\alpha$ : and we have thus a simple $\grave{a}$ posteriori verification of the form in which, several years ago, I presented Bezout's Method of Elimination.

2, Stone Buildings, W.C., March 5, 1863. 